Georgia State University

ScholarWorks @ Georgia State University

\title{
The effects of cognitive defusion and thought distraction on emotional discomfort and believability of negative self-referential thoughts
}

Akihiko Masuda

Georgia State University, amasuda@gsu.edu

M. P. Twohig

A. R. Stormo

A B. Feinstein

Y. Chou

See next page for additional authors

Follow this and additional works at: https://scholarworks.gsu.edu/psych_facpub

Part of the Psychology Commons

\section{Recommended Citation}

Masuda, Akihiko; Twohig, M. P.; Stormo, A. R.; Feinstein, A B.; Chou, Y.; and Wendell, J. W., "The effects of cognitive defusion and thought distraction on emotional discomfort and believability of negative selfreferential thoughts" (2010). Psychology Faculty Publications. 96.

https://scholarworks.gsu.edu/psych_facpub/96

This Article is brought to you for free and open access by the Department of Psychology at ScholarWorks @ Georgia State University. It has been accepted for inclusion in Psychology Faculty Publications by an authorized administrator of ScholarWorks @ Georgia State University. For more information, please contact scholarworks@gsu.edu. 
Authors

Akihiko Masuda, M. P. Twohig, A. R. Stormo, A B. Feinstein, Y. Chou, and J. W. Wendell

This article is available at ScholarWorks @ Georgia State University: https://scholarworks.gsu.edu/psych_facpub/96 
The paper is accepted for publication in Journal of Behavior Therapy and Experimental Psychiatry

Running head: COGNITIVE DEFUSION

The Effects of Cognitive Defusion and Thought Distraction on Emotional Discomfort and Believability of Negative Self-Referential Thoughts

\author{
Akihiko Masuda*, ** \\ Georgia State University \\ Michael P. Twohig \\ Utah State University
}

Analia R. Stormo, Amanda B. Feinstein, Ying-Yi Chou, \& Johanna W. Wendell Georgia State University

*Address editorial correspondence to: Aki Masuda

Department of Psychology

Georgia State University

P.O. Box 5010

Atlanta, GA 30302-5010

Phone: (404) 413-6298

Fax: (404) 413-6207

psyaxm@langate.gsu.edu

**A copy of the complete intervention manual is available from the first author. 


\begin{abstract}
Previous research has shown that rapid vocal repetition of a one-word version of negative self-referential thought reduces the stimulus functions (e.g., emotional discomfort and believability) associated with that thought. The present study compares the effects of that defusion strategy with thought distraction and distraction-based experimental control tasks on a negative self-referential thought. Non-clinical undergraduates were randomly assigned to one of three protocols. The cognitive defusion condition reduced the emotional discomfort and believability of negative self-referential thoughts significantly greater than comparison conditions. Favorable results were also found for the defusion technique with participants with elevated depressive symptoms.
\end{abstract}

Key Words: acceptance; Acceptance and Commitment Therapy; believability; cognitive defusion; emotional discomfort; mindfulness; self-referential thoughts; thought distraction 


\section{Introduction}

In recent years, acceptance- and mindfulness-based interventions (see Hayes, Follette, \& Linehan, 2004) have been widely discussed and studied in the field of cognitive behavior therapy (CBT). As a group, these therapies generally focus more on the functional effects of private events (e.g., thoughts, feelings, physiological sensations, memories) than on content and frequency of these events in understanding and treating psychopathology (e.g., Fisher \& Wells, 2005; Linehan, 1993; Segal, Teasdale, \& Williams, 2004). An example of these interventions is Acceptance and Commitment Therapy (ACT) (Hayes, Strosahl, \& Wilson, 1999).

ACT includes various techniques to change the function of private events in order to promote psychological health (e.g., Hayes, Luoma, Bond, Masuda, \& Lillis, 2006). One set of techniques used particularly for this purpose are cognitive defusion strategies (see Luoma \& Hayes, 2009). Cognitive defusion is roughly conceptualized as altering the literal meaning and behavior-regulatory function of private events without necessarily altering the form, frequency, or situational sensitivity of these events (Blackledge, 2007). In ACT, defusion techniques are often employed in contexts where clients are excessively entangled or fused with their private events, such as negative self-referential thought (e.g., "I am depressed”; “I” = “depression”).

Control-based strategies, such as avoidance, thought suppression, and distraction are conventional coping methods for unwanted private events (Hayes, Wilson, Gifford, Follette, \& Strosahl, 1996). Research has shown that these strategies, which directly target the form and frequency of unwanted private events, may be ineffective and potentially paradoxical (e.g., Eifert \& Heffner, 2003; Gutiérrez, Luciano, Rodríguez, \& 
Fink, 2004). Among these, the iatrogenic effect is especially clear in the case of suppression methods (e.g., Feldner, Zvolensky, Eifert, \& Spira, 2003; Levitt, Brown, Orsillo, \& Barlow, 2004; Marcks \& Woods, 2007). For example, in a study by Marcks and Woods, 2005 participants instructed to suppress their personally relevant intrusive thoughts were found to report difficulty in doing so and increased distress after suppression attempt. For this reason, ACT aims at strengthening the process of cognitive defusion for difficult private events, instead of employing control-based coping strategies.

Several focused studies have investigated the effects of specific cognitive defusion techniques on negative self-referential thoughts (e.g., Healy et al., 2008; Masuda, Hayes, Sackett, \& Twohig, 2004; Masuda et al., 2009). One study investigated the effects of a commonly used cognitive defusion technique, "I am having a thought that...” (Healy et al., 2008). The study suggests that negative self-referential statements (e.g., "my life is pointless") when presented in the defused format (e.g., "I am having a thought that my life is pointless") can decrease the emotional discomfort associated with that statement and increase willingness to be exposed to these statements.

Another study examined the effects of rapid vocal repetition of a one-word version of a negative self-referential thought—another commonly used defusion technique (Masuda et al., 2004). This technique is derived from the observation that when a word is rapidly repeated out loud, the context required for the word to have its literal meaning is altered, and the literal meaning of the word disappears (Titchener, 1910). In the study, the defusion protocol consisted of a defusion rationale, training, and 30-second rapid vocal repetition of a one-word version of the negative self-referential thought. The defusion condition was compared to a distraction-based experimental control task 
(reading an article on Japan) and thought control task. Primary dependent variables were reductions in emotional discomfort and believability of the self-referential negative thoughts. Results revealed that the defusion condition decreased the stimulus functions (i.e., emotional discomfort and believability) associated with these thoughts more so than comparison conditions across all participants.

A subsequent group parametric study examined whether the duration of one-word thought repetition systematically altered the impact of this defusion strategy (Masuda et al., 2009). The reduction of emotional discomfort was found to bottom out after 3 to 10 seconds of rapid repetition, whereas the maximum reduction of believability occurred after 20 to 30 seconds of repetition. These findings additionally suggest that the actual experiential exercise of rapid thought repetition is crucial for altering the stimulus function of negative self-referential thoughts, and that emotional discomfort and believability may be distinctive functional aspects of cognitive events.

While interesting and encouraging, previous studies did not clearly reveal the relative effects of rapid thought repetition. Although the original defusion study (Masuda et al., 2004) reported the superiority of the defusion condition to comparison conditions, multiple treatment interference likely occurred because each participant in the study received multiple interventions. Additionally, the thought-control condition in the study was employed for controlling gross demand characteristics. The subsequent parametric study (Masuda et al., 2009) did not compare the defusion protocol with an active comparison condition, either.

Additionally, the effects of this cognitive defusion technique have not been examined in clinical or sub-clinical samples. To date, there are several focused 
experimental studies reporting the positive effects of an acceptance-based emotion regulation strategy in clinical samples with emotional disorders (Campbell-Sills, Barlow, Brown, \& Hofmann, 2006; Liverant, Brown, Barlow, \& Roemer, 2008). These results are relevant to the present study in part because the acceptance-based strategy shares aims with defusion strategies (i.e., changes in the stimulus function of aversive private events). Given the lack of empirical evidence, it is worthwhile to investigate the effects of defusion using a subgroup from a non-clinical sample, such as non-clinical college undergraduates who report elevated psychological symptoms.

As a response to these emerging questions, the present study investigates the relative impact of the cognitive defusion technique (i.e., rapid thought repetition) on a self-referential negative thought, as compared to a thought distraction strategy. The present study had several methodological and conceptual advantages over previous defusion studies (Masuda et al., 2004; Masuda et al., 2009). The study employed a group design format in order to reduce extraneous variables, such as multiple treatment interference, and it more clearly identified the active comparison condition. The thought distraction strategy, which is roughly defined as an effort of selectively attending to an emotionally less distressing event or situation (Gross, 1998), was selected as the active comparison condition for several reasons. First, a distraction technique is a defined control strategy (e.g., McCaul \& Malott, 1984) that appears theoretically distinct from cognitive defusion because of its primary focus on reducing the frequency of an unwanted private event by shifting attention away from it. Second, distraction can be an appropriate active comparison condition because it has been found effective in some contexts, especially in the situations of mildly and moderately aversive events (e.g., 
Gutiérrez et al., 2004). Finally, the distraction-based experimental control condition (e.g., reading an article about Japan) was also added to the study as a control group in order to control non-specific factors. Based on previous research findings (e.g., Gutiérrez et al. Masuda et al., 2004; Masuda et al., 2009), it was hypothesized that the cognitive defusion condition would reduce the emotional discomfort and believability of negative selfreferential thoughts greater than the comparison conditions. It was further predicted that positive effects of cognitive defusion also would be seen among participants who reported “elevated depressive symptoms.”

2. Method

\subsection{Participants and Settings}

The study was conducted at a large public 4-year university in Georgia. Participants were 132 students $\left(77 \%, n_{\text {Female }}=102\right)$ recruited from undergraduate psychology courses through a web-based research participant pool. The age of the participants ranged from 17-60 years $(M=20.91, S D=6.96)$. The ethnic composition of the sample was diverse with 39\% $(n=50)$ identifying as "European American," 33\% ( $n=$ 43) identifying as “African American,” 14\% $(n=19)$ identifying as “Asian American/Pacific Islander American,” 9\% $(n=11)$ identifying as "Hispanic American,” and 5\% ( $n=7)$ identifying as "other” or "bicultural."

\subsection{Demographic and Screening Form}

Following the consent procedure, participants completed a demographic form and the BDI-II. The demographic variables included gender, age, and ethnicity.

\subsubsection{Beck Depression Inventory II.}


The Beck Depression Inventory-II (BDI-II) (Beck, Steer, \& Brown, 1996) is often used as a screening form for general psychological functioning. The measure appeared to be particularly relevant because of the link between negative self-referential thoughts (the dependent variables of the study) and depression (the event measured by the BDI-II). The BDI-II is a 21-item self-report questionnaire that is designed to assess recent depressive symptoms. Each item is rated using a 4-point severity scale, ranging from 0 to 3 . The total score ranges from 0 to 63 with greater scores suggesting greater depressive symptoms. The BDI-II has shown good test-retest reliability $(r=.93)$ and has demonstrated a high correlation with the original BDI ( $r=.93$, Beck et al, 1996). In the present study, the mean BDI-II score was used as the cutoff for selecting a sub-sample of participants with elevated depressive symptoms.

\subsection{Thought Selection and Assessment}

Following completion of the demographic form and the BDI-II, thought selection and assessment was conducted. Thought selection and assessment were administered by research investigators who were trained by the first author. The procedure and instruction of the thought selection and procedure was closely scripted. The participant was given an assessment form and orally instructed to generate one negative self-referential thought that had entered the participant's mind repeatedly and regularly and that had been found particularly disturbing and believable (e.g., “I am not pretty”). Participants were then asked to restate the thought in one word (e.g., "ugly”). The degree of emotional discomfort and the believability of the thought were assessed using a 100-mm Likertstyle visual analog scale before and immediately after the intervention. Responses ranged from 0 (not at all uncomfortable) to 100 (very uncomfortable) for the discomfort scale, 
and from 0 (not at all believable) to 100 (very believable) for the believability scale. If participants could not come up with a thought that was above 50 on the discomfort or 50 on believability scales, they were prompted to identify another negative self-referential thought that was more uncomfortable and believable. Participants, who could not come up with a thought that was above 50 on the discomfort or 50 on believability scales after the prompt, were eliminated in order to fit participants to the purpose of the present investigation. Participants were not informed of the inclusion criteria.

\subsection{Procedure}

Participants were randomly assigned to one of three conditions: (a) cognitive defusion, (b) thought distraction, and (c) distraction-based experimental control (control) tasks. All of these intervention conditions were 5 minutes long and closely scripted. Investigators ran participants in all conditions to minimize experimenter effects. Experimenters were trained by the first author who is a licensed clinical psychologist and has extensive research experience. A weekly research meeting was held to ensure adherence to the scripted intervention.

Both active interventions were equal in (a) components, (b) duration (5-minutes), (c) sequence of components, (d) contents of training (e.g., the use of the word "milk" highlighting the use of the assigned strategy), and (e) the number of prompts given during the intervention phase. A distraction-based experimental control condition was added to the current work to control non-specific factors, such as the duration of the contact with the experimenter.

\subsubsection{Cognitive Defusion Condition.}


The cognitive defusion task consisted of the defusion rationale, defusion training, and a 30-second rapid vocal repetition of the one-word target thought. The rationale and training were drawn from the original ACT book (Hayes et al., 1999). The defusion rationale identified the positive aspects of human verbal behavior (e.g., thinking, language), but also addressed the role of language and thinking in human suffering. The participant was told that a distressing self-referential thought may be relatively automatic and that people often identify themselves with the literal content of their thoughts. To exemplify this, defusion training was introduced where the participant was initially asked to say the word "milk" once and to notice all of its perceptual functions (e.g., "white," “cold,” “creamy”). The participant was then instructed to repeat the word "milk" out loud as rapidly as possible for 20 seconds. The participant was told to notice what happens to the perceptual functions during the word repetition. Participants typically reported that the meaning of the word began to disappear, and most participants noted that more direct functions appeared (e.g., “The word milk did not mean anything.” "It became just a sound.”). The experimenter then suggested that this defusion experience could be applicable to the participant's self-referential negative thought, indicating that negative thoughts are also simply sounds with conventional meanings. The participant was then asked to repeatedly state the one-word self-referential negative thought (e.g., "fat”) aloud as fast as possible until informed to stop. The experimenter said, "stop" after 30 seconds passed. As in Masuda et al. (2004), to maintain engagement in this condition, the experimenter provided a verbal prompt (i.e., "faster" and "louder") to the participant after 10 and 20 seconds.

\subsubsection{Thought Distraction Condition.}


The thought distraction condition consisted of a thought distraction rationale, distraction training, and the actual attempt at distracting from the target negative selfreferential thought. The thought distraction condition began with a statement suggesting that cognitions cause and trigger actions and emotions, and that negative thoughts are the source of human suffering, followed by a statement suggesting that distracting oneself from negative thoughts by thinking of something different is a solution. After the brief rationale, the participant received thought distraction training using the word, "milk." Similar to the defusion condition, the participant was asked to say the word "milk" once and to notice all of its perceptual functions (e.g., "white,” “cold,” "creamy”). Then, the participant was instructed not to think of the word "milk" by thinking of something emotionally neutral or less unpleasant for about 20 seconds. After briefly discussing the usefulness and credibility of the distraction strategy on the word "milk," the experimenter then suggests applying this experience to his or her negative self-referential thought in order to prevent psychological suffering. Prior to the actual use of the thought distraction strategy on the target thought, the participant was first asked to say the one-word version of that thought once to focus on the target thought. The participant was then instructed to distract from the target negative self-referential thought by thinking of something else until the experimenter said "stop.” As in the defusion condition, in order to maintain engagement in this condition, the experimenter provided a verbal prompt (i.e., "don’t think about it") to the participant after 10 and 20 seconds. The experimenter said "stop" after 30 seconds passed.

Because of the potentially greater variation of actual distraction strategies and the quality of such attempts relative to the cognitive defusion condition, a brief experimental 
manipulation check was conducted for the thought distraction group at post-experiment. Participants were asked what they had tried to think about during distraction, and how successful they were at it using a 3- point scale, ranging from 0 (not being able to do so at all), 1 (somewhat being able to do so), to 2 (being able to do so well). Examples of the distracting thought contents included upcoming pleasant events (e.g., Thanksgiving, a friend's birthday), something neutral, such as thinking about the next scheduled appointment, and stimuli in the experimental room, such as the door or window. Even though the content varied, the attempts fit the conceptualization of thought distraction as an effort of selectively attending to an emotionally less distressing event or situation (Gross, 1998). The average rating was $1.35(S D=.66)$, falling in the range between somewhat able and very well able to distract themselves from the target thoughts.

\subsubsection{Distraction-based Experimental Control Condition.}

The experimental control condition (control condition) did not include a rationale. The condition involved reading an emotionally neutral article about Japan (i.e., vacations to the mountains) for 5 minutes.

\section{Results}

\subsection{Characteristics of Sample}

The mean score for the 132 participants on the BDI-II, which was used to assess participants’ general psychological functioning at pre, was $9.09(S D=7.64)$. The score fell in the average range of a non-clinical population. Of the 132 participants who were randomly assigned to the cognitive defusion $(n=41)$, thought distraction $(n=48)$, or control condition $(n=43), 15$ participants (defusion $=5$, thought distraction $=6, \&$ control $=4$ ) were excluded due to extraneous methodological factors. Specifically, 13 of 
these participants were excluded because of difficulty in identifying a negative selfreferential thought or inability to follow instructions. Two participants (one from each active condition) discontinued their participation prior to the completion of assessment because they expressed elevated levels of frustration and became argumentative toward the experimenter. Furthermore, an additional 14 participants (defusion $=5$, thought distraction $=4$, \& distraction-based experimental control $=5$ ) were excluded at the time of data analyses because of the inclusion criteria (i.e., 50 or greater in both emotional discomfort and believability at pre-intervention). Results were equivalent when the data of the 14 participants were included in the following data analyses.

As a result, 103 participants with relatively high degrees of emotional discomfort and believability were left for data analyses; cognitive defusion $(n=31)$, thought distraction ( $n=38)$, and distraction-based experimental control condition $(n=34)$. Of those, 76\% were females $(n=78)$. The age of the participants ranged from 17-60 years $(M=21.17, S D=7.72)$. The final sample was ethnically diverse with $38 \%(n=39)$ identifying as “European American,” 30\% $(n=31)$ identifying as “African American,” 17\% ( $n=17)$ identifying as “Asian/Pacific Islander,” 11\% $(n=11)$ identifying as “Hispanic American,” and 4\% $(n=4)$ identifying as “other” or “bicultural.”

\subsection{Pre-intervention Group Differences \& the Effects of Potential Confounding Factors}

Chi-square testes and ANOVAs revealed that the groups did not differ in the compositions of gender and ethnicity background. BDI-II score, the age of participants, and pre-intervention emotional discomfort and believability $(p>.10)$, did not differ significantly by group. Furthermore, results of two 4 (i.e., experimenters) by 2 (i.e., time) repeated measures ANOVAs revealed no main effect of experimenter or interaction of 
time and experimenter in the emotional discomfort or believability of negative selfreferential thoughts, $F s<1.17$, $p s>.32$.

\subsection{Effects on Self-Referential Negative Thoughts}

The means, standard deviations, and effect sizes of emotional discomfort and believability scores for all conditions are presented in Table 1 . The results for the emotional discomfort and believability of the negative self-referential thoughts were analyzed separately, using two 3 (condition: cognitive defusion, thought distraction, \& control condition) by 2 (time: pre- and post-intervention) repeated measure ANOVAs.

\subsubsection{Emotional Discomfort.}

Results showed a main effect for time, $F(1,100)=129.16, p<.001$, and a two way interaction between condition and time, $F(2,100)=5.15, p<.01$ (see Figure 1 ). The interaction was decomposed both by looking at the effects of time across each condition and by looking at the effects of condition at each time point. Emotional discomfort at post-intervention was found to be significantly lower than pre-intervention emotional discomfort across all conditions ( $F$ 's $>15$, ps < .001). Pairwise comparisons revealed that at post, the defusion group reported significantly lower levels of emotional discomfort than the thought distraction group $(p<.05)$ and distraction-based experimental control group $(\mathrm{p}<.001)$. The thought distraction group reported significantly lower emotional discomfort than the experimental control group $(p<.05)$.

\subsubsection{Believability.}

Almost identical results were found in the believability of the negative selfreferential thoughts with one exception. In the believability of negative self-referential 
thought, no group difference between thought distraction and experimental control groups were found at post-intervention $(p>.05)$.

\subsection{Effects on Individuals with Elevated Depressive Symptoms}

The mean score of BDI-II in 103 participants was $9.9(S D=8.18)$. As a result, using the BDI-cut off score of 10, 42 participants were selected as participants with elevated depressive symptoms; cognitive defusion $(n=14)$, thought distraction $(n=10)$, and distraction experimental control condition $(n=18)$. An ANOVA revealed that BDI-II score did not significantly differ by group $(p>.19)$.

\subsubsection{Emotional Discomfort.}

The means, standard deviations, and effect sizes of emotional discomfort and believability scores for all conditions among those with elevated depressive symptoms are presented in Table 2. Given a significant group difference of emotional discomfort at pre-intervention, an analysis of covariance (ANCOVA) on post-intervention emotional discomfort, covarying the pre-treatment levels of emotional discomfort was performed. Results revealed the main effect of condition, $F(2,38)=4.73, p<.05$. Pairwise comparisons revealed that the cognitive defusion group had significantly lower emotional discomfort than the experimental control condition $(p<.01)$ Pairwise comparisons did not reveal other significant group differences.

\subsubsection{Believability.}

A 3 (condition: cognitive defusion, thought distraction, \& control condition) by 2 (time: pre- and post-intervention) repeated measure ANOVA revealed the main effect for time, $F(1,100)=129.16, p<.001$. Pairwise comparisons revealed a significant reduction of believability at post-treatment as compared to pre-treatment $(p<.001)$. The time by 
condition interaction effect was not found to be significant, $F(2,39)=2.57, p=.089$. However, at post-intervention, a medium effect size $(d=-.65)$ was found in the comparison between cognitive defusion and thought distraction conditions, and a large effect size $(d=-.99)$ was found in the comparison between cognitive defusion and experimental control conditions. Both of these results favored the cognitive defusion condition.

\subsection{Exploratory Analyses on the Role of Depressive Symptoms}

Because similar patterns were found between the overall participant group and the subgroup with elevated depressive symptoms, the roles of depressive symptom (i.e., BDIII scores) on negative self-referential thoughts and the effects of intervention were further investigated. A correlational analysis, using the overall sample of 103 participants, revealed that the depressive symptom (BDI scores) was not a predictor of emotional discomfort $(r=.06, p=.53)$ or believability $(r=.08, p=.44)$ at post-intervention. Subsequently, a hierarchical regression analysis was conducted separately for each postintervention outcome to investigate depressive symptom as a moderator of the relations between intervention and that outcome variable. In the analysis, the variable of interest at pre-intervention was entered into the first step, followed by depressive symptom in the second step. Subsequently, intervention condition (i.e., categorized as $1=$ defusion, $2=$ thought distraction, \& $3=$ control condition) was entered in the third step. Finally, depressive symptom and the interaction term were entered in the third step. Results revealed that depressive symptom was not found to be a moderator of the relations between intervention and post-intervention emotional discomfort $(\beta=-.02, t=-.08, p$ 
$=.93)$ or between intervention and post-intervention believability $(\beta=-.16, t=-.70, p$ $=.49)$.

\section{Discussion}

Consistent with previous cognitive defusion studies (Masuda et al., 2004; Masuda et al., 2009), the present study suggests that the rapid repetition of a one-word version of a self-referential negative thought, combined with a clinical rationale and training, reduces the emotional discomfort and believability of that thought at least temporarily. The present investigation is the first to show the superior effects of the defusion procedure over a thought distraction strategy, a control-based coping method that is commonly employed by clients prior to seeking treatment. Exploratory analyses suggest that depressive symptoms do not predict outcome variables at post-intervention or moderate the relation between the present interventions and these post-intervention outcomes. The defusion protocol was also found to effectively reduce the believability and emotional discomfort associated with negative self-referential thought among individuals with elevated depressive symptoms. In conclusion, the study suggests that, regardless of the levels of depressive symptom, the cognitive defusion technique is an effective strategy for altering the stimulus function of negative self-referential thought in a non-clinical sample. Given this encouraging finding, it may be worthwhile to investigate whether the present positive finding is generalized to a clinical sample with depression in future research.

Further research is needed to clarify the difference between cognitive defusion and thought distraction strategies due to the lack of functional distinctions between the two active strategies. The present study revealed that although the effects of thought 
distraction were smaller than those of the cognitive defusion condition, its effects on emotional discomfort and believability of negative self-referential thoughts appeared significant compared to the control condition. These findings were somewhat surprising because a distraction strategy and cognitive defusion technique were thought to be functionally different from each other.

Several speculations can be drawn regarding the positive effects of thought distraction. One speculation is that so-called acceptance and mindfulness strategies, such as cognitive defusion, are not fundamentally different from control-based coping strategies, although its aims are said to be fundamentally unique (process- and functionfocused). As some experts in CBT (e.g., Hoffmann \& Asmundson, 2008) suggest, the differences might be simply the matter of degree.

Alternatively, the lack of the functional distinction was in part due to the nature of the negative self-referential thoughts. More specifically, the effects of the two strategies may be moderated by the severity of target private events. Literature on pain tolerance has shown that a distraction strategy is an effective coping strategy for mild or moderate pains, but not for severe ones (McCaul \& Malott, 1984). A previous comparison experiment between an acceptance strategy and distraction strategy on pain tolerance revealed that both interventions were equally effective in a lower pain context, but that the superiority of an acceptance-based condition emerged in the context of greater pain intensity (Gutierrez et al., 2004). Given these previous findings, it was speculated that the lack of functional distinction between the two active conditions in the present study might have been in part because of negative self-referential thoughts that were not disturbing enough. In fact, integrity checks revealed that the majority of participants in 
the thought distraction condition were able to perform the distraction strategy. This line of reasoning suggests that it is worthwhile to compare the two strategies, using a clinical sample, which is thought to experience more severely negative self-referential thoughts. It is also possible that the lack of a functional difference between defusion and distraction conditions can be attributed to the inadequacy of the believability measure. Using the believability measure, the current study attempted to capture the degree to which a thought is experienced as a mental event, regardless of the literal content of the thought (Hayes et al., 2006). However, it was extremely likely that participants viewed the believability measure as a scale of "how true or valid the content of the thought is." One suggestion for future research is to change the anchor and verbal instruction of believability scale, from "how believable (true) is the thought?" to "how much do you experience the thought simply as a mental event, rather than as an absolute fact about you?” Another suggestion is to employ an alternative acceptance- and process-oriented measure, such as "how OK is it for you to have this thought?" in order to distinguish it from a content-based believability measure (i.e., veracity of thought). These alternative measures may be sensitive enough to capture the process of cognitive defusion.

Another notable finding is that much greater variability (SDs) of discomfort and believability scores were seen at post-intervention than at pre-intervention across all intervention groups. These results clearly reveal that there were larger inter-individual differences at post-intervention, and suggest that the manipulations had varying effects across participants. It is speculated that the variability might have been due to preintervention levels of discomfort and believability, participants' coping repertoire for 
difficult thoughts (e.g., coping strategies), demand characteristics, participants’ psychological characteristics, learning history, and other unknown factors.

The present study has other conceptual and methodological limitations. The active conditions in the present study consisted of multiple components, and it is unclear which components or combination of components is responsible for changes in the stimulus function of the negative self-referential thoughts. However, given the results of other focused studies (e.g., Masuda et al., 2009; McMullen et al., 2008; Takahashi, Muto, Tada, \& Sugiyama, 2002), the experiential part of an acceptance exercise seems to be crucial in the functional change of private events. The present study also did not examine the impact of actual repetition of the target thought alone, although research on semantic satiation (Esposito \& Pelton, 1971) suggests that word repetition without a rationale is unlikely to produce favorable effects.

Similar to many focused experimental studies, follow-up assessments were not included in this investigation. Although this can be considered a limitation, the long-term effects of this particular defusion technique may not be as important as showing that the process is useful in particular contexts. The purpose of defusion exercises is not to alter or defuse the meaning or function of target thoughts permanently. In fact, the meaning and function of a given thought is contextually determined and it is hoped that the client can maintain some level of contextual control over the meaning and function of all thoughts. Defusion exercises simply help the client experience that the emotional impact/meaning, with which negative self-referential thoughts typically accompany, is not absolute or inherent, and that a negative self-referential thought can be experienced as it is as a thought, rather than as what it says it is (Hayes et al., 1999). For this reason, the 
effects of the present defusion exercise, and other defusion techniques, need not be longlasting, and yet they still provide clients with a new experience and thereby new learning. Methodologically, relative to the cognitive defusion condition, the thought distraction condition still had greater variability. During the intervention phase, the participants in the distraction condition were instructed to perform the distraction strategy by thinking about something different. While the participants received the identical instructions, the content of the private events used for the distraction strategy varied across participants. Although the manipulation check administered for the thought distraction condition suggested that the condition served its purpose, additional methodological control in the thought distraction strategy (i.e., pre-determining the stimulus used for distraction) seems warranted. It is also important to note that the verbal prompt used for the distraction condition during the actual exercise (i.e., “don’t think about it!”) appeared more closely related to a suppression strategy than a distraction method.

Another less systematized area of the study was the use of multiple experimenters. Although a closely scripted intervention was employed and statistical analyses did not reveal a significant effect of experimenter, variations in the characteristics of experimenters, such as their interaction style with participants were highly likely. The audio-taped or video-taped recording of intervention sessions is recommended for adherence checks in future research.

Furthermore, to minimize the variability in each intervention condition, the use of a computerized program may be useful as an alternative mode of experimental procedure for future research. The present investigation used a contact-based format in order to 
maintain a therapeutic atmosphere in an analogue research setting. However, a more standardized procedure, such as the use of a computerized program or audio-taped intervention, may be important to systematically manipulate the variables of interests.

Finally, perhaps the major limitation of the present study is the exclusive reliance on self-report measures. From an ACT perspective, discomfort and believability of private events are functional processes, and they should be studied within the context of ongoing stimulus-behavior relations. Self-report type methods do not measure these processes directly when they occur. It is difficult to directly assess the stimulus function of the negative self-referential thought, thus, the development of behavioral methodology that captures the function of self-referential thoughts seems extremely important.

In sum, despite these limitations, the present investigation is the first study that shows the superior effects of the defusion procedure over a thought distraction strategy, a commonly used coping strategy by psychotherapy clients. The present findings are encouraging, and further investigations on the process and effects of a cognitive defusion approach seem warranted. 


\section{References}

Beck, A.T., Steer, R. A., \& Brown, G. K. (1996). Beck Depression Inventory-Second Edition (BDI-II). San Antonio, TX: The Psychological Corporation.

Blackledge, J. T. (2007). Disrupting verbal process: Cognitive defusion in acceptance and commitment therapy and other mindfulness-based psychotherapies. The Psychological Record, 57, 555-576.

Campbell-Sills, L., Barlow, D. H., Brown, T. A. \& Hofmann (2006). Effects of suppression and acceptance on emotional responses of individuals with anxiety and mood disorders. Behaviour Research and Therapy, 44, 1251-1263.

Eifert, G. H. \& Heffner, M. (2003). The effects of acceptance versus control contexts on avoidance of panic-related symptoms. Journal of Behavior Therapy and Experimental Psychiatry, 34, 293-312.

Esposito, N. J., \& Pelton, L. H. (1971). Review of the measurement of semantic satiation. Psychological Bulletin, 75, 330-346.

Feldner, M. T., Zvolensky, M. J., Eifert, G. H., \& Spira, A. P. (2003). Emotional avoidance: an experimental test of individual differences and response suppression using biological challenge. Behaviour Research and Therapy, 41, 403-411.

Fisher, P. L., \& Wells, A. (2005). Experimental modification of beliefs in obsessivecompulsive disorder: A test of the metacognitive model. Behaviour Research and Therapy, 43, 821-829.

Gross, J. J. (1998). The emerging field of emotion regulation: An integrative review. Review of General Psychology, 2, 271-299. 
Gutiérrez, O., Luciano, C., Rodríguez, M., \& Fink, B. C. (2004). Comparison between an acceptance-based and a cognitive-control-based protocol for coping with pain. Behavior Therapy, 35, 767-784.

Hayes, S. C., Barnes-Holmes, D., \& Roche, B. (Eds.). (2001). Relational Frame Theory: A Post-Skinnerian account of human language and cognition. New York: Plenum Press.

Hayes, S. C., Follette, V. M., \& Linehan, M. M. (Eds.) (2004). Mindfulness and acceptance: Expanding the cognitive behavioral tradition. New York: Guilford Press.

Hayes, S. C., Luoma, J., Bond, F., Masuda, A., \& Lillis, J. (2006). Acceptance and Commitment Therapy: Model, processes, and outcomes. Behaviour Research and Therapy, 44, 1-25.

Hayes, S. C. Strosahl, K. D., \& Wilson, K. G. (1999). Acceptance and Commitment Therapy: An experiential approach to behavior change. New York: Guilford Press.

Hayes, S. C., Wilson, K. G., Gifford, E. V., Follette, V. M., \& Strosahl, K. (1996). Emotional avoidance and behavioral disorders: A functional dimensional approach to diagnosis and treatment. Journal of Consulting and Clinical Psychology, 64, 1152-1168.

Healy, H., Barnes-Holmes, Y., Barnes-Holmes, D., Keogh, C., Luciano, C., \& Wilson, K. (2008). An experimental test of a cognitive defusion exercise: Coping with negative and positive self-statements. The Psychological Record, 58, 623-640. 
Hoffmann, S. G., \& Asmundson, G. J. (2008). Acceptance and mindfulness-based therapy: New wave or old hat? Clinical Psychology Review, 28, 1-16.

Levitt, J. T., Brown, T. A., Orsillo, S. M., \& Barlow, D. H. (2004). The effects of acceptance versus suppression of emotion on subjective and psychophysiological response to carbon dioxide challenge in patients with panic disorder. Behavior Therapy, 35, 747-766.

Linehan, M. M. (1993). Cognitive-behavioral treatment of borderline personality disorder. New York: Guilford.

Liverant, G. I., Brown, T. A., Barlow, D. H., \& Roemer, L. (2008). Emotion regulation in unipolar depression: The effects of acceptance and suppression of subjective emotional experience on the intensity and duration of sadness and negative affect. Behaviour Research and Therapy, 46, 1201-1209.

Luoma, J. B., \& Hayes, S. C. (2009). Cognitive defusion. In W. O’Donohue \& J. E. Fisher(Eds.), General principles and empirically supported techniques of cognitive behavior therapy. (pp. 181-188). Hoboken, NJ: John Wiley \& Sons.

Marcks, B. A., \& Woods, D. W. (2005). A comparison of thought suppression to an acceptance-based technique in the management of personal intrusive thoughts: A controlled evaluation. Behaviour Research and Therapy, 43, 433-445.

Masuda, A., Hayes, S. C., Sackett, C. F., \& Twohig, M. P. (2004). Cognitive defusion and self-referential negative thoughts: Examining the impact of a ninety year old technique. Behaviour Research and Therapy, 42, 477-485. 
Masuda, A., Hayes, S. C., Twohig, M. P., Drossel C., Lillis, J., \& Washio, Y. (2009). A parametric study of cognitive defusion and the believability and discomfort of negative self-referential thoughts, Behavior Modification, 33, 250-262.

McCaul, K. D., \& Malott, J. M. (1984). Distraction and coping with pain. Psychological Bulletin, 95, 516-533.

McMullen, J., Barnes-Holmes, D., Barnes-Holmes, Y., Stewart, I., Luciano, C., \& Cochrane, A. (2008). Acceptance versus distraction: Brief instructions, metaphors and exercises in increasing tolerance for self-delivered electric shocks. Behaviour Research and Therapy, 46, 122-129.

Segal, Z. V., Teasdale, J. D. \& Williams, J. M. G. (2004). Mindfulness-based cognitive therapy: Theoretical rationale and empirical status. In S. C. Hayes, V. M. Follette, \& M. M. Linehan (Eds.), Mindfulness and acceptance: Expanding the cognitive behavioral tradition (pp. 45-65). New York: Guilford Press.

Takahashi, M., Muto, T., Tada, M., \& Sugiyama, M. (2002). Acceptance rationale and increasing pain tolerance: Acceptance-based and FEAR-based practice. Japanese Journal of Behavior Therapy, 28, 35-46.

Titchener, E. B. (1910). A text-book of psychology. New York: MacMillan. 
Table 1

Average Scores, Standard Deviations, and Effect Sizes of Emotional Discomfort and Believability of Negative Self-Referential Thoughts by Condition and Time

\begin{tabular}{|c|c|c|c|c|c|c|}
\hline \multirow[b]{2}{*}{ Conditions: } & \multicolumn{3}{|c|}{ Emotional Discomfort } & \multicolumn{3}{|c|}{ Believability } \\
\hline & Pre & Post & $\begin{array}{l}\text { Pre-Post } \\
\text { within } d\end{array}$ & Pre & Post & $\begin{array}{l}\text { Pre-Post } \\
\text { within } d\end{array}$ \\
\hline 1. Cognitive Defusion $(n=31)$ & $\begin{array}{c}77.65 \\
(13.25)\end{array}$ & $\begin{array}{c}38.81 \\
(24.76)\end{array}$ & 1.70 & $\begin{array}{c}73.16 \\
(14.49)\end{array}$ & $\begin{array}{c}39.10 \\
(27.69)\end{array}$ & 1.60 \\
\hline 2. Thought Distraction $(n=38)$ & $\begin{array}{c}78.03 \\
(11.04)\end{array}$ & $\begin{array}{c}52.17 \\
(24.89)\end{array}$ & 1.39 & $\begin{array}{c}80.11 \\
(14.44)\end{array}$ & $\begin{array}{c}59.11 \\
(26.34)\end{array}$ & 1.47 \\
\hline 3. Control $(n=34)$ & $\begin{array}{l}75.50 \\
(9.89)\end{array}$ & $\begin{array}{c}63.29 \\
(18.70)\end{array}$ & 1.36 & $\begin{array}{c}79.41 \\
\text { (16.33) }\end{array}$ & $\begin{array}{c}70.62 \\
(24.70)\end{array}$ & 1.60 \\
\hline Between Condition Cohen's $d$ & & & & & & \\
\hline Condition 1 vs. Condition 2 & & -.54 & & & -.74 & \\
\hline Condition 1 vs. Condition 3 & & -1.13 & & & -1.20 & \\
\hline Condition 2 vs. Condition 3 & & -.51 & & & -.45 & \\
\hline
\end{tabular}


Table 2

Average Scores, Standard Deviations, and Effect Sizes of Emotional Discomfort and Believability of Negative Self-Referential Thoughts and Beck Depression Inventory-II by Condition and Time among Participants with Elevated Depressive Symptom

\begin{tabular}{|c|c|c|c|c|c|c|c|}
\hline \multirow[b]{2}{*}{ Conditions: } & \multicolumn{3}{|c|}{ Emotional Discomfort } & \multicolumn{3}{|c|}{ Believability } & \multirow[t]{2}{*}{ BDI-II } \\
\hline & Pre & Post & $\begin{array}{l}\text { Pre-Post } \\
\text { within } d\end{array}$ & Pre & Post & $\begin{array}{l}\text { Pre-Post } \\
\text { within } d\end{array}$ & \\
\hline 1. Cognitive Defusion & 83.71 & 43.36 & 1.90 & 77.21 & 42.21 & 1.30 & 20.74 \\
\hline$(n=14)$ & $(11.10)$ & (28.87) & & (13.60) & (27.11) & & $(8.42)$ \\
\hline 2. Thought Distraction & 82.90 & 52.60 & 1.16 & 80.80 & 52.60 & .61 & 17.70 \\
\hline$(n=10)$ & $(10.16)$ & (32.85) & & $(17.42)$ & (33.18) & & $(8.51)$ \\
\hline 3. Control & 76.00 & 64.00 & .87 & 81.33 & 67.94 & .67 & 15.38 \\
\hline$(n=18)$ & (6.88) & (15.44) & & $(16.25)$ & (24.79) & & $(4.80)$ \\
\hline \multicolumn{8}{|c|}{ Between Condition Cohen's $d$} \\
\hline Cond. 1 vs. Cond. 2 & & -.30 & & & -.65 & & \\
\hline Cond. 1 vs. Cond. 3 & & -.93 & & & -.99 & & \\
\hline Cond. 2 vs. Cond. 3 & & -.47 & & & -.22 & & \\
\hline
\end{tabular}

Note: BDI-II = Beck Depression Inventory-II; Cond. = Condition 


\section{Figure Caption}

Figure 1. Means of emotional discomfort and believability of negative self-referential thoughts at pre and post by condition. 

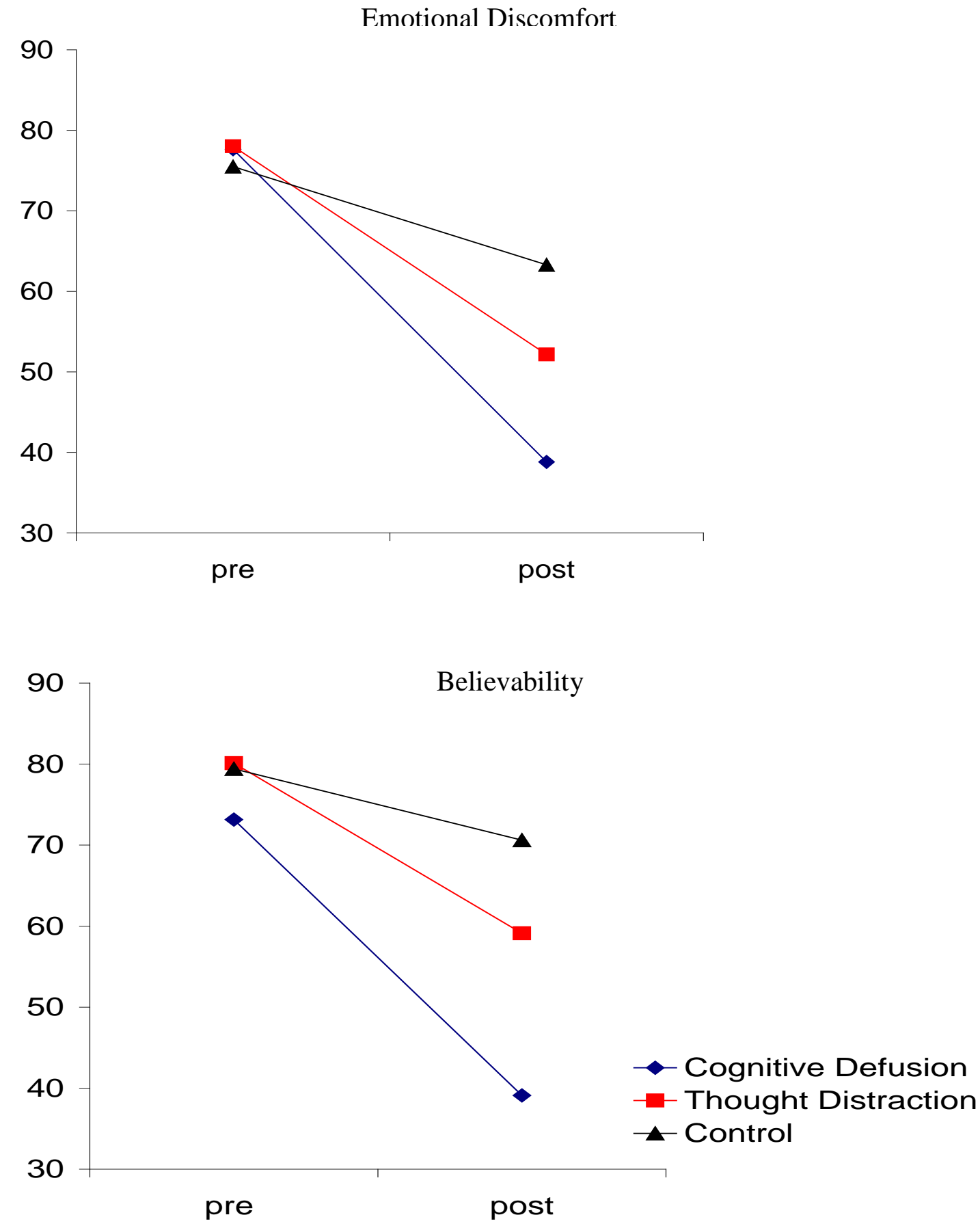